\title{
Previous Motor Actions Outweigh Sensory Information in Sensorimotor Statistical Learning
}

\author{
Barbara Feulner, ${ }^{1,2}$ Danilo Postin, ${ }^{1,3}$ Caspar M. Schwiedrzik, ${ }^{4,5,6}$ and Arezoo Pooresmaeili, ${ }^{1,6}$
}

\author{
https://doi.org/10.1523/ENEURO.0032-21.2021
}

${ }^{1}$ Perception and Cognition Lab, European Neuroscience Institute Göttingen-A Joint Initiative of the University Medical Center Göttingen and the Max-Planck-Society, Göttingen 37077, Germany, ${ }^{2}$ Bioengineering Department, Imperial College London, London SW7 2BU, United Kingdom, ${ }^{3}$ Department of Psychiatry, School of Medicine and Health Sciences, Carl von Ossietzky University of Oldenburg, Bad Zwischenahn 26160, Germany, ${ }^{4}$ Neural Circuits and Cognition Lab, European Neuroscience Institute Göttingen-A Joint Initiative of the University Medical Center Göttingen and the Max-PlanckSociety, Göttingen 37077, Germany, ${ }^{5}$ Perception and Plasticity Group, German Primate Center-Leibniz Institute for Primate Research, Göttingen 37077, Germany, and ${ }^{6}$ Leibniz ScienceCampus Primate Cognition, Göttingen 37077, Germany

\begin{abstract}
Humans can use their previous experience in form of statistical priors to improve decisions. It is, however, unclear how such priors are learned and represented. Importantly, it has remained elusive whether prior learning is independent of the sensorimotor system involved in the learning process or not, as both modality-specific and modality-general learning have been reported in the past. Here, we used a saccadic eye movement task to probe the learning and representation of a spatial prior across a few trials. In this task, learning occurs in an unsupervised manner and through encountering trial-by-trial visual hints drawn from a distribution centered on the target location. Using a model-comparison approach, we found that participants' prior knowledge is largely represented in the form of their previous motor actions, with minimal influence from the previously seen visual hints. By using two different motor contexts for response (looking either at the estimated target location, or exactly opposite to it), we could further compare whether prior experience obtained in one motor context can be transferred to the other. Although learning curves were highly similar, and participants seemed to use the same strategy for both response types, they could not fully transfer their knowledge between contexts, as performance and confidence ratings dropped after a switch of the required response. Together, our results suggest that humans preferably use the internal representations of their previous motor actions, rather than past incoming sensory information, to form statistical sensorimotor priors on the timescale of a few trials.
\end{abstract}

Key words: learning; prior; probabilistic; representation; saccade; sensorimotor

\section{Significance Statement}

Humans can learn statistical regularities and later use them as priors to inform decisions. It remains unclear what type of representation is used to store and integrate past experience. We designed an experiment where humans had to combine visual information over multiple trials to locate a hidden target location. Using computational modeling, we found that participants represented past experience in the form of their previous decisions, and not directly by memorizing the visual cues. As a consequence of overweighing past decisions relative to the veridical visual information, gained experience did not generalize across two different contexts, albeit they differed minimally with respect to the prior. Hence, the process through which past experience is learned determines its influence on our decisions.

Received January 25, 2021; accepted June 23, 2021; First published August 19, 2021.

The authors declare no competing financial interests.
Author contributions: B.F., C.M.S., and A.P. designed research; B.F. and D.P. performed research; B.F. analyzed data; B.F., C.M.S., and A.P. wrote the paper. 


\section{Introduction}

We often have to make decisions based on sparse and uncertain sensory information. Previous research has shown that in these cases humans use Bayesian inference where the current sensory information (likelihood) and the previously acquired knowledge (priors) are integrated, each weighted by their respective uncertainty (Körding and Wolpert, 2004; Wei and Körding, 2010). While the majority of previous studies have examined whether the perceptual and sensorimotor decisions follow the rules of a Bayesian framework (Ernst and Banks, 2002; Körding and Wolpert, 2004), less emphasis has been placed on understanding how likelihoods and especially statistical priors are learned and represented in the first place. A number of elegant recent studies have tried to bridge this gap by investigating how people learn likelihoods (Sato and Kording, 2014) and priors (Berniker et al., 2010) to perform Bayesian computations. Interestingly, the timescale of the two types of learning varied vastly, with fast learning of likelihood but slow learning of prior distributions. It remains unknown why such an asymmetry should exist, as theoretically both types of learning are equivalent. It has been hypothesized that learning about the likelihood versus learning about the prior involves different neural mechanisms, potentially hinting to the fact that their respective distributions might be represented in different regions of the brain (Vilares et al., 2012).

Learning of statistical priors is itself not a uniform process as it shows dependencies on the specific context where the learning occurs. In the Bayesian framework, priors are a form of abstract knowledge (Tenenbaum et al., 2006, 2011; Battaglia et al., 2013), which can be generalized across different contexts. However, previous findings regarding the generalization of statistical priors have been mixed. Some studies have shown that statistical learning of priors is very narrow-band and context/modality specific in perceptual (Frost et al., 2015) and sensorimotor (Hewitson et al., 2018; Yin et al., 2019) domains, thus preventing learned information to transfer to different contexts/modalities. Other studies, on the other hand, provided evidence for generalization (Sato and Kording, 2014; Kiryakova et al., 2020), although generalization, in some instances, seemed to occur differently for different parameters of a statistical distribution, e.g., the mean and the variance of a distribution (Fernandes et al., 2014). The finding that some aspects of learning could generalize, while others could not, was confirmed by a

This work was initially supported by a seed fund Grant from Leibniz Science Campus Primate Cognition, Göttingen, Germany (C.M.S. and A.P.) and continued by the European Research Council Starting Grant 716846 (to A.P.). C.M.S. was supported by the German Research Foundation Emmy Noether Grant SCHW1683/2-1.

Acknowledgements: We thank Tomke Schoss for her help with the pilot study.

Correspondence should be addressed to Barbara Feulner at b.feulner18@ imperial.ac.uk or Arezoo Pooresmaeili at a.pooresmaeili@eni-g.de.

https://doi.org/10.1523/ENEURO.0032-21.2021 Copyright $@ 2021$ Feulner et al.

This is an open-access article distributed under the terms of the Creative Commons Attribution 4.0 International license, which permits unrestricted use, distribution and reproduction in any medium provided that the original work is properly attributed. recent study showing that, for instance, in Bayesian time estimation, priors can be generalized across stimuli but not motor actions (Roach et al., 2017).

Therefore, despite an increasing number of studies testing generalization and transfer, the exact rules determining generalizability remain unclear. One potential reason for these seemingly contradictory results is a lack of a formal definition of what is learned. It has been argued that when learning is not generalized, a policy (i.e., a specific rule for action) rather than knowledge (i.e., abstract and context-independent information) is acquired through learning (Chambers et al., 2019). However, it is not clear what features of the learning dynamics determine whether a policy or knowledge is acquired during encounters with the learned information.

To investigate learning and generalizability of a prior distribution, we employed a saccadic eye movement task similar to the design of a previous study (Dekleva et al., 2016), where participants had to learn to locate a hidden target. The location of the hidden target corresponded to the mean of a circular normal distribution. In each trial, a visual hint sampled from the underlying distribution was shown and participants indicated their current estimate with a saccadic eye movement, looking either toward (pro-saccade) or to the exact opposite direction of the estimate (anti-saccade). To successfully estimate the hidden target location, participants had to combine information across multiple trials. This design allowed us to investigate whether participants formed their prior knowledge by combining the visual information or by combining the previous motor actions across time, under different saccadic response contexts. Our results from two experiments indicate that sensorimotor learning of a spatial prior in both response contexts is largely guided by previous motor plans, rather than by previous sensory input in form of visual hints. Despite the high degree of similarity of pro-saccades and anti-saccades in their learning behavior, suggesting a motor-independent learning algorithm, the learned prior in one context did not generalize to the other. We propose that the lack of transfer between the two contexts is a natural consequence of their shared learning algorithm in which previous motor actions outweigh sensory information.

\section{Materials and Methods}

In this study, we report the results of two experiments investigating how a spatial prior is learned under different oculomotor response contexts. The second experiment was identical to the first and served as a control for ensuring that participants were aware of how the location of the learned prior varied across blocks of the experiment (see the description of experiment 1 and experiment 2). We therefore describe the methods common to both experiments and mention the differences where they apply.

\section{Participants}

In total, 41 participants were recruited for this study; 21 participants (age range 22-38 years, main $=26.05$, $\mathrm{SD}=3.80,10$ females) took part in the first experiment. All participants had normal $(N=9)$ or corrected-to-normal 
A

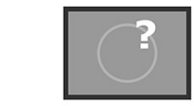

Hidden Target Task (HT)

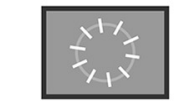

Calibration Task (CT)

C
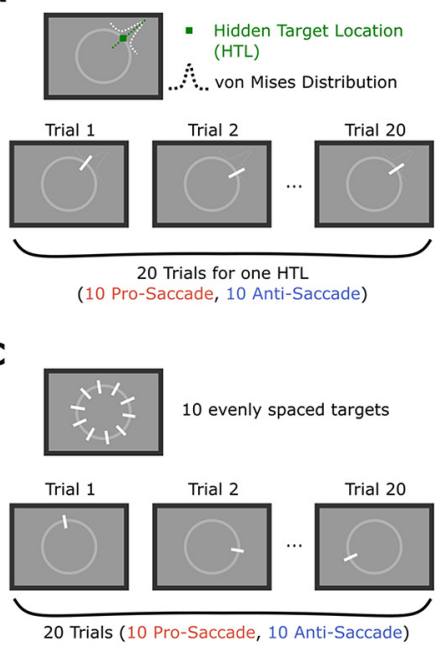

B

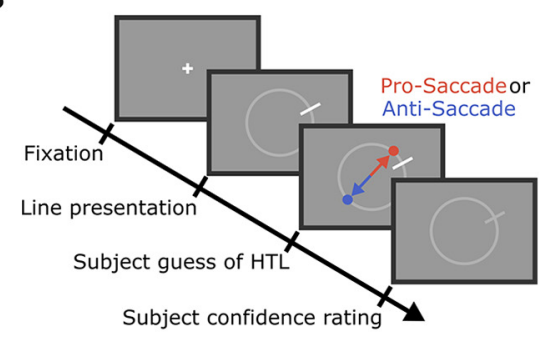

D

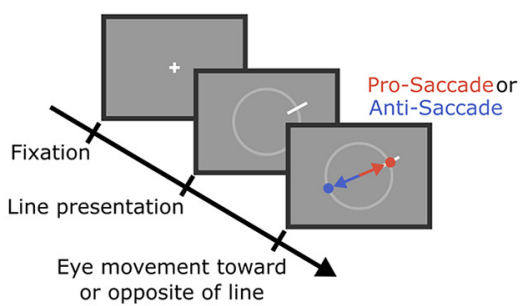

E

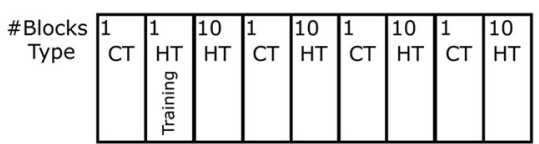

$\mathbf{F}$

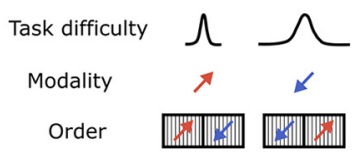

Figure 1. Experimental design of the hidden target task employed to study the statistical learning of a spatial prior in two different visuo-motor contexts. $\boldsymbol{A}, \boldsymbol{B}$, Main task of the experiment. $\boldsymbol{A}$, Participants were told to estimate the location of a hidden treasure on a ring by observing and combining information provided by the visual hints across trials. The hidden target location was defined as the mean of a von Mises distribution and the hints, presented at each trial, were samples drawn from this underlying distribution. Participants had 20 trials to estimate the location of the hidden target, after which a new hidden target had to be found. Participants used their gaze to indicate their responses. B, Each trial started with a fixation period, after which the hint was presented, and participants had to indicate their guess about the location of the hidden target by either looking at it (pro-saccade response) or by looking exactly opposite to it (anti-saccade response). In half of the trials (i.e., consecutive 10 trials), participants had to use prosaccades, and in the other half they used anti-saccades, with a randomized order across blocks. C, $\boldsymbol{D}$, Calibration task used to estimate the motoric error of each participant for pro-saccades and anti-saccades. Participants had to directly look either at the lines (pro-saccade response) or exactly opposite to the lines (anti-saccade response). $\boldsymbol{E}$, Block-design of the experiment. $\boldsymbol{F}$, We compared learning across two levels of difficulty and two different response types. Task difficulty was varied by changing the concentration of the von Mises distribution (compare Materials and Methods). Finally, we tested whether knowledge could be transferred from one visuo-motor context to the other. For this, we also varied the order of pro-saccade and anti-saccade responses across blocks.

vision $(N=12)$. One participant was excluded from the data of the first experiment as the postexperiment questionnaire indicated that the participant had misunderstood the task. A total of 20 participants (age range 23-38 years, main $=28.95, S D=5.07,11$ females) took part in the second experiment (with no exclusion) and all had normal $(N=16)$ or corrected-to-normal vision $(N=4)$. Participants were recruited from the general population of the city of Göttingen, Germany, using flyer and online advertisement and received cash financial compensation for their participation. Participation was voluntary; all participants were informed about the study procedure and gave written consent before the test session. The study was approved by the local ethics committee of the Universitätsmedizin Göttingen (UMG), under the proposal number 15/7/15.

\section{Experimental setup}

The stimuli were presented at the center of a calibrated ViewPixx/EEG monitor (VPixx Technologies; dimension: $53 \times 30 \mathrm{~cm}$, refresh rate: $120 \mathrm{~Hz}$ ) with a resolution of $1920 \times 1080$ pixels at a viewing distance of $60 \mathrm{~cm}$. All experiments were scripted in MATLAB, using Psychophysics toolbox (Brainard, 1997). Eye movements were measured using the Eyelink1000+ eye tracking system (SR Research) in a desktop mount configuration, recording the right eye, with a sampling rate of $1000 \mathrm{~Hz}$. A chin rest was used to stabilize the participant's head. The EyeLink camera was controlled by the EyeLink toolbox in MATLAB (Cornelissen et al., 2002). At the beginning of each experiment, as well as after every 10 blocks of the hidden target task (see experiment 1), the eye tracking system was calibrated using a 13-point standard EyeLink calibration procedure. Calibration was repeated until an average error of maximum 0.5 degrees of visual angle (dva) was achieved, and the error of all points was below $1 \mathrm{dva}$. If the calibration accuracy dropped during the experiment, e.g., because of the subjects' movement, the experimenter recalibrated the eye tracking system again.

\section{Experiment 1}

The experiment comprised two tasks: a calibration task (to estimate the motor error of pro-saccades and antisaccades) and the main task, referred to as the "hidden target task" (Fig. 1). There were in total four blocks of the 
calibration task ( $n=20$ trials in each block) and 40 blocks of the hidden target task ( $n=20$ trials in each block). Each experiment started with a block of the calibration task followed by one training block for the hidden target task ( $n=10$ trials in this block). The data from this training phase was not analyzed. Thereafter, the experiment proceeded to the main task where participants performed 10 blocks of the hidden target task followed by one block of the calibration task. This sequence was repeated four times (Fig. 1E).

\section{Hidden target task}

Participants were instructed to look for a "hidden treasure" location on a ring with a radius of $7.5 \mathrm{dva}$, centered in the middle of the screen (Fig. 1A). The word hidden treasure was used in our instructions to the participants to make the task more realistic and engaging, however we will refer to the task as the hidden target task throughout. Each trial started with a fixation period, where participants had to fixate for $0.5 \mathrm{~s}$ on the white cross (size $=0.1875$ dva, color: white, displayed on a half-gray background) in the middle of the screen (Fig. 1B). After that, a white line (length $=1.125 \mathrm{dva}$, color: white) was presented and participants had $3 \mathrm{~s}$ to estimate the hidden target location for this trial and indicate their guess either by looking at it (pro-saccade) or by looking opposite of it (anti-saccade) and fixate their estimated location for $0.5 \mathrm{~s}$. Thereafter, participants rated their level of confidence in their guess on a discrete scale from 1 to 6 , where 1 means very uncertain and 6 means very certain about the target location. The confidence rating had to be done within $4 \mathrm{~s}$ by pressing the corresponding key on the keyboard (keys S, D, F, J, K, L corresponding to confidence level 1-6, respectively).

Participants were told that they had 20 trials to guess the location of the hidden target, after which a new hidden target had to be found. To estimate the hidden target location participants had to closely monitor the location of a line that was presented in every trial and served as a visual hint. The hidden target location was the mean of a von Mises distribution and each visual hint was a sample drawn from this distribution (Dekleva et al., 2016). Hence, by paying attention to the location of the hint across trials, participants were able to infer the underlying hidden target location. Participants indicated their estimates by looking either at where they thought the hidden target was located on the ring (pro-saccade), or at a location directly opposite to it (anti-saccade). Ten consecutive trials of a block of 20 trials required pro-saccade responses, and the other 10 anti-saccade responses. Importantly, the location of the hidden target in a block of 20 trials stayed the same and did not change after the switch in the required response type. The type of the required response (either pro-saccade or anti-saccade) was visually indicated by an instruction display presented every 10 trials. Participants were instructed to perform the same type of response for 10 trials in a row, until the response type changed.

In total, there were 40 hidden target blocks. The target location of each block, which is the mean of the von Mises distribution, was randomly drawn from a fixed set of 20 locations evenly distributed on the circle. Thus, each location only appeared twice during the experiment. To familiarize the participants with the connection between the hints and the hidden target location, 10 training trials were performed in the beginning of each experiment. After the 10 training trials, participants saw all the 10 lines together on the screen, as well as the correct hidden target location. Furthermore, after each hidden target block, participants saw where the actual hidden target location was, but they did not receive feedback about their performance on a trial-by-trial basis. As such, in our experiments, learning was unsupervised.

Four different experimental conditions, counter-balanced across blocks, were tested. Each block was either easy or hard, controlled by adapting the concentration of the von Mises distribution, and either ordered with first pro-saccade then anti-saccade, or first anti-saccade then pro-saccade response (Fig. 1F). For the easy task condition, the concentration of the von Mises distribution (defined by $\kappa$ which is a measure of dispersion, where $1 / \kappa$ is equivalent to the variance $\sigma^{2}$ dist of the distribution) was 30 $\left(\sigma_{\text {dist }} \sim 10^{\circ}\right)$, for the hard task condition it was $5\left(\sigma_{\text {dist }} \sim 26^{\circ}\right)$ and for the training it was $80\left(\sigma_{\text {dist }} \sim 6^{\circ}\right)$. As the concentrations of these distributions are relatively large, we could treat the von Mises distribution as a normal distribution and use standard statistics.

\section{Calibration task}

The aim of this task was to quantify the participant-specific motor error of the visually driven pro-saccades and anti-saccades. Each block of this task consisted of 20 trials, from which the first 10 were pro-saccades and the last 10 were anti-saccades. On each trial, one out of 10 equally distributed locations on the circle (same circle as in the hidden target task) were selected and a target line was presented at that location (Fig. 1C). Participants were instructed to look either directly at the displayed line (prosaccade in the first 10 trials), or directly opposite to where it appeared (anti-saccades, second 10 trials). Additionally, it was highlighted that this task is completely independent of the hidden target task. Each trial consisted of an initial fixation phase, where participants had to fixate on the white cross in the middle of the screen for $0.5 \mathrm{~s}$. After that, a white line appeared and participants had to either look at it or opposite of it (Fig. 1D). In the beginning of a block of 10 trials, participants received an instruction display indicating whether they had to perform pro-saccades or anti-saccades during the upcoming trials.

\section{Successful response}

For both tasks, a successful response was defined as follows. Participants had to move their gaze from the central fixation point toward a peripheral location on the ring. As soon as the gaze moved away from the fixation point by more than a radius of $5.375 \mathrm{dva}$, a successful response was possible. To complete the response, participants furthermore had to hold their gaze on their intended landing position for $0.5 \mathrm{~s}$. During online measurements, as soon as the gaze crossed the 5.375-dva threshold, we calculated the mean and standard deviation of the gaze data in a moving window of $0.5 \mathrm{~s}$. Whenever the standard deviation of the last $0.5 \mathrm{~s}$ of the gaze data fell below $1 \mathrm{dva}$, the 
A

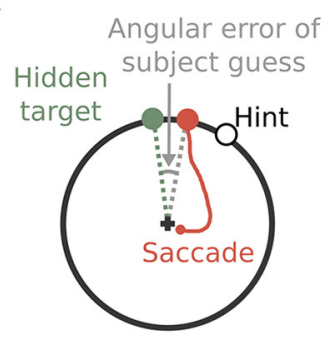

C

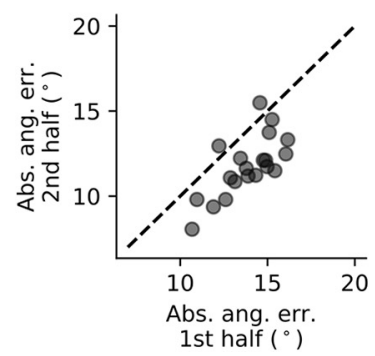

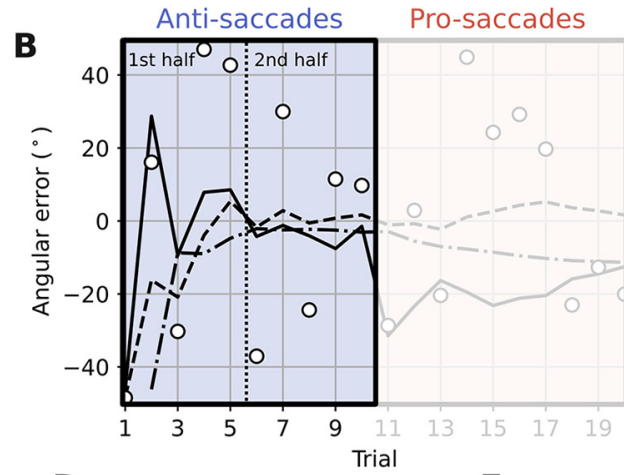

$\mathbf{E}$ $\begin{array}{ll}\text {-- Subject guess } \\ \text {-- } & \text { Cum. avg. hints } \\ \text {-.- } & \text { Cum. avg. guesses } \\ \text { - } & \text { Hint }\end{array}$
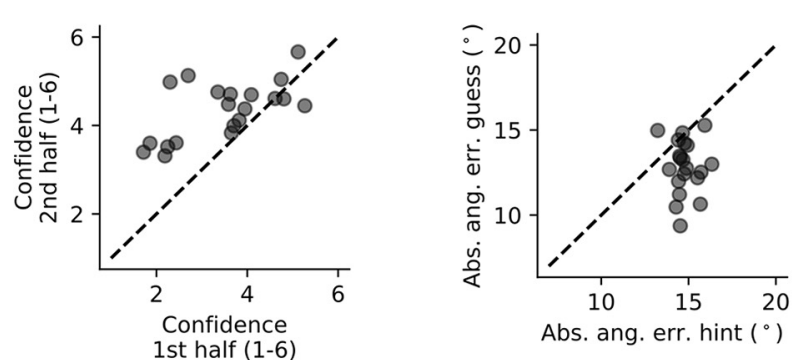

Figure 2. Participants successfully accumulate information and learn on a short time scale. $\boldsymbol{A}$, The angular difference between the participant's guess and the true location of the hidden target was used to measure learning. $\boldsymbol{B}$, Example block. To test learning, we compared the performance in trials 1-5 (first half) to the performance in trials $6-10$ (second half). $\boldsymbol{C}$, The absolute angular error in the second half is lower than in the first half (paired $t$ test: $t=7.25, p<0.0001, N=20$ ). $\boldsymbol{D}$, Participants' confidence is higher in the second half than in the first half (paired $t$ test: $t=-4.39, p=0.0003, N=20$ ). $\boldsymbol{E}$, The absolute angular error of participants is lower than the absolute angular error of the visual hints, i.e., participants' guesses are closer to the center of the von Mises distribution compared with the presented visual hints (paired $t$ test: $t=4.92, p=0.0001, N=20$ ).

gaze shift from the fixation point toward a peripheral location was considered as a successful response in that trial.

\section{Experiment 2}

The second experiment served as a control that participants had indeed understood the task structure. Specifically, we aimed to ensure that participants were aware that the target location stayed the same across all 20 trials of a block of the hidden target task and did not change after a switch of response modality in the same block (i.e., after trial 10). To this end, we used the same experimental design and procedures as in experiment 1 with the exception of the following modifications: (1) we slightly modified the instruction slides during the experiment to enforce the notion that all 20 trials within one block belonged to the same hidden target location and did not change after a switch in response modality; and (2) we asked the participants after each block to report whether they were aware that all 20 trials belonged to the same hidden target location. They had to press a button to indicate their response, either yes or no.

\section{Data analysis}

\section{Data preprocessing}

The recorded raw eye movement data were transformed to MATLAB files by using a MATLAB library for eye movement analysis (Manohar, 2019). Participants' estimates in each trial were calculated offline by averaging the eye movement data of the last $100 \mathrm{~ms}$, out of the total $500 \mathrm{~ms}$ necessary for a successful response. No further post hoc constraints on gaze data were used to identify successful responses beyond the method used online and described above (Successful response). The main eye movement parameter that we analyzed was the angular distance between the participant's estimate and the true hidden target location (Fig. 2A). Failed trials were excluded from the analysis. A trial could fail because of three reasons. First, there could have been a disturbance with the eye tracking system or the participant's calibration so that the gaze position was not correctly detected, which made it necessary to re-calibrate the eye tracker. Second, the participant could have been too slow to indicate their guess in time $(3 \mathrm{~s})$. Third, to exclude erroneous pro- instead of anti-saccades and vice versa, we analyzed the distribution of angular errors and found a bimodal distribution. We set a threshold at $100^{\circ}$, which separated both modes. Thus, every trial with an absolute angular error bigger than $100^{\circ}$ was categorized as failed because of the wrong response type. Number of failed trials because of the first two reasons were 12/800 and 13/800 in pro and anti of the calibration task, and 150/4000 and $132 / 4000$ in pro and anti of the hidden target task, respectively. Based on the third reason, 59/800 and $43 / 800$ were marked as failed in pro and anti of the calibration task and $104 / 4000$ and $196 / 4000$ in pro and anti of the hidden target task. Hence, less than $<9 \%$ of trials of each condition were excluded from further analysis. 


\section{Statistical analysis}

Statistical analyses were done using $\mathrm{R}$ and Python. To fit the linear regression models, we either used the Im function in $\mathrm{R}$ or the OLS function from the python package statsmodels. To evaluate learning we used paired, twosided $t$ tests to compare several parameters within a participant. In these cases (Fig. 2), the assumption of normality was tested by applying a Shapiro-Wilk test to the data. We did not use circular statistics as subjects' responses were highly localized on the ring (Fig. $3 A, B$ ). Learning was assessed by measuring the decrease in the absolute angular error between the participant's estimate and the true hidden target location across trials.

To compare the performance difference between prosaccades and anti-saccades, we calculated a "modality difference index" (Fig. $3 C$ ). For this, we first calculated the mean of the absolute angular error for pro-saccade and anti-saccade response trials. The modality difference index is then given by the difference between the two means, divided by their sum (calculated per subject). We started our analysis by only using the data from the first 10 trials of each block (Figs. 1-4). Only when the transfer between modalities was examined, we used all 20 trials of each block (Figs. 5, 6).

\section{Theoretical bounds for learning performance}

To evaluate participants' performance, we computed the theoretical lower bounds on the absolute angular error they could potentially achieve by using all the information that was available to them. Participants could use the previously seen visual hints and the memory of their previous motor actions (referred to as previous guesses) to infer the most probable location of the target on each trial. These sources of information are error prone since on each trial participants had only seen a limited number of visual hints (i.e., sampling error) and their previous responses contained motoric noise. We assumed that these two sources of error are independent. The error because of the limited number of visual hints was calculated as the cumulative mean of all hints seen so far, which represents an optimal way of combining samples over time to estimate the mean of a distribution. A constant motor error, measured for each participant during the calibration task, was used to represent the motoric noise. The variance of the joint estimate, derived from combining visual hints and motor actions, was then calculated by adding the variance of the two sources, based on the assumption of their independence. To understand how participants used different sources of information to make decisions on a trial-by-trial basis, we employed a detailed modelcomparison approach as described below.

\section{Modeling participants' behavior}

We used linear regression models (Im package in $\mathrm{R}$ ) to analyze the single subject behavior. To this end, the angular error of participants' estimate in a given trial (i.e., their guess in trial $t$, Guess $s_{t}$ ) was fitted by models having a diverse set of independent variables as shown in Table 1.

The five single predictor models we tested were (1) using the visual hint in each trial; (2) using the visual hint in the preceding trial (t-1); (3) using the cumulative average of all visual hints so far; (4) using the guess from the previous trial; and (5) using the cumulative average of all previous guesses (Fig. 4A; Table 1). The dependent variable was the angular error of a participant's estimate in a certain trial $\left(\right.$ Guess $\left._{t}\right)$. The independent variable was the angular error of the estimate, given by one of the abovementioned strategies. In a second stage, we tested linear regression models with multiple independent variables. For this, we included previous guesses from up to three time steps in the past, as well as the visual hints from the current and up to three time steps in the past. Our choice of including three trials in the past was inspired by a naive model search approach (Extended Data Fig. 4-2), which indicated that including up to three trials in the past provided the best fit to the participants' data. Thus, in our multipredictor models only the data from trial four to trial 10 was included, allowing all models to be tested on the same data. Each of the described regression models was fitted to the single subject data using R's Im package. To compare the models, we calculated $\mathrm{BIC}, \delta \mathrm{BIC}$, and Bayesian weights (Burnham and Anderson, 2004), to assess the likelihood of each model being the best fit to the data (cf below, Evaluating model performance). Since these values are normalized, they can be used to determine the model that on average best fits the participants' data.

\section{Evaluating model performance}

To compare the different linear regression models presented above we used a model comparison evaluation based on Bayesian weights (Burnham and Anderson, 2004). For this, we first calculated BIC values for each model. Each $\mathrm{BIC}$ value was rescaled by calculating $\triangle \mathrm{BCl}$, which is calculating the difference to the smallest BIC value in the group of models considered. This forces the best model to have $\triangle \mathrm{BCl}=0$ and the other models to have positive values. We then calculated Bayesian weights $\omega$, as described in (Burnham and Anderson, 2004). The Bayesian weights of all tested models in Figure $4 B$ sum up to 1 and define the probability of being the best model, among the one tested.

\section{Results}

To investigate the dynamics of sensorimotor statistical learning of a spatial prior and its dependence on the response modality, we designed an experiment where participants had to find a hidden target and indicate their guess by either a pro-saccade or an anti-saccade. Participants learned the location of each hidden target within 20 trials, of which a block of 10 trials required prosaccades and the other block of 10 trials required antisaccades as the response modality (Fig. $1 A$ ). This design allowed us to probe whether the learning dynamics shows dependencies on the response modality, hence being modality specific. We also used another task, referred to as the calibration task, to estimate each participant's motoric noise during the visually driven execution of pro-saccades or anti-saccades. In contrast to the calibration task, in the hidden target task the main error source is the uncertainty regarding the hidden target location. As the same motor system is used in both the hidden 


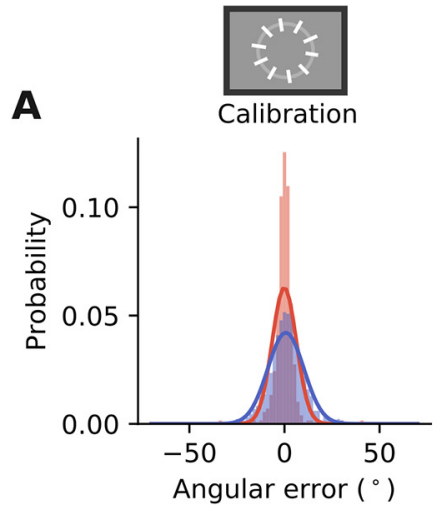

D

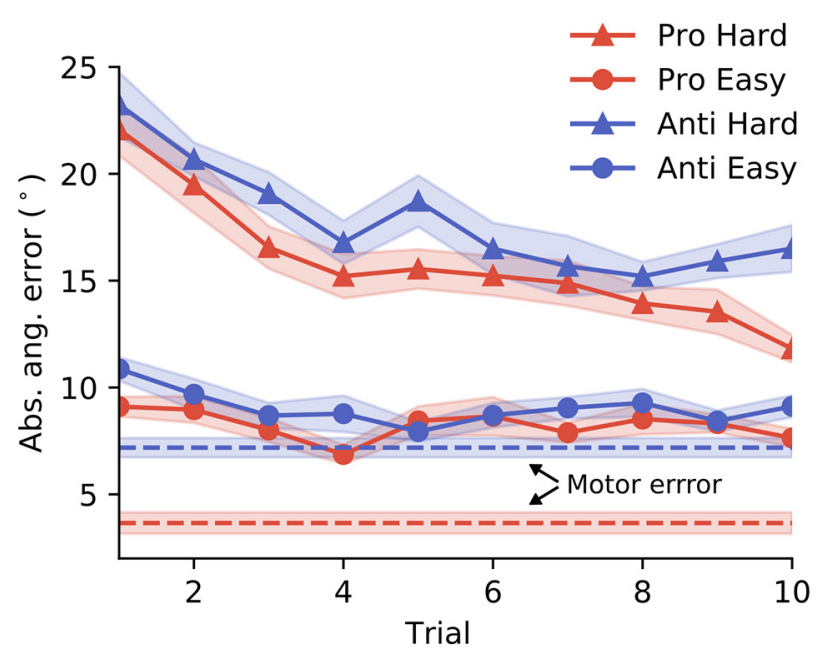

$\mathbf{F}$

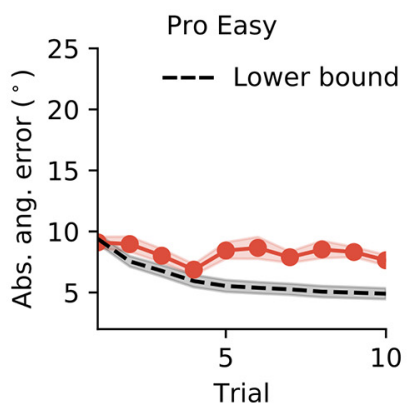

H

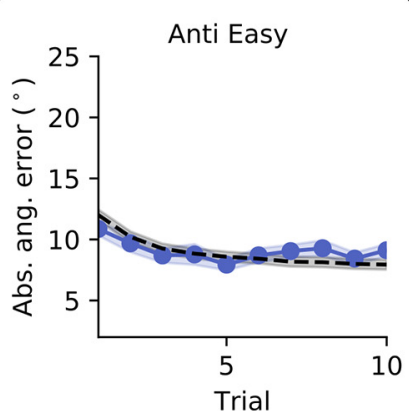

B

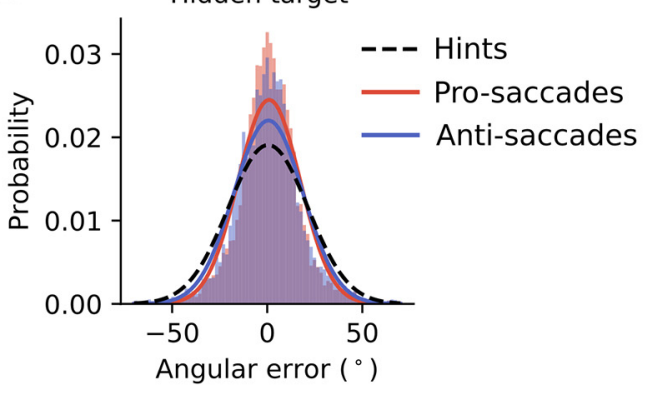

C

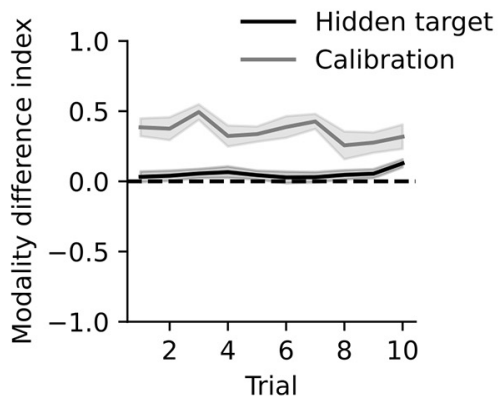

E

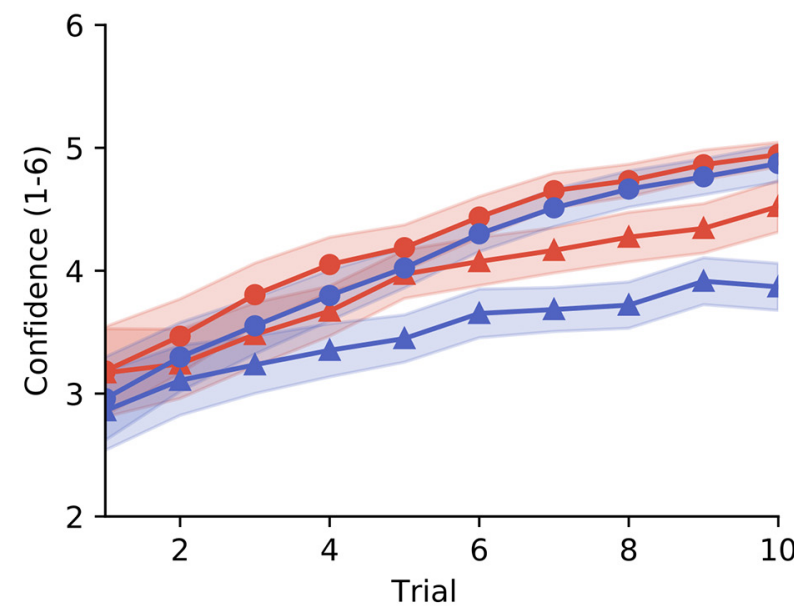

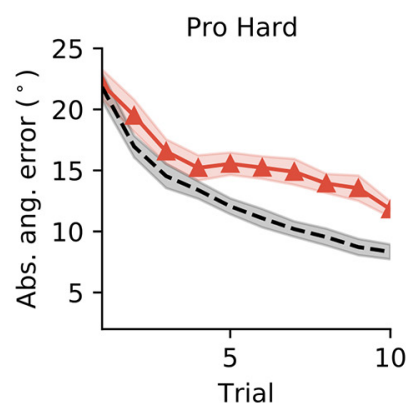

J

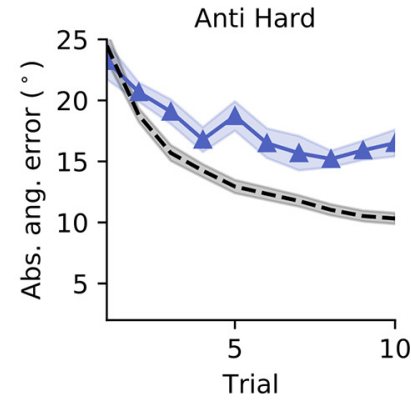

Figure 3. Similarity of learning curves across response modalities. $\boldsymbol{A}$, The distribution of the angular error for pro-saccade and antisaccade response in the calibration task. $\boldsymbol{B}$. The distribution of the angular error for pro-saccade and anti-saccade response in the hidden target task. $\boldsymbol{C}$, The modality difference index quantifies the difference between the absolute angular error in pro-saccade and anti-saccade trials. The shaded area indicates the SEM. $\boldsymbol{D}$, Time course of the absolute angular error for each of the four different conditions (two response types $\times$ two difficulties). Here and in the following panels, except stated otherwise, shaded areas represent the SEM $(N=20)$. $\boldsymbol{E}$, Time course of the confidence ratings for each of the four different conditions. $\boldsymbol{F}$-J, Participants' learning curves compared with the lower bound. The lower bound is given by taking the cumulative average of all hints presented so far and adding the error because of motoric noise, estimated from the calibration task.

target task as well as the calibration task, we assumed that the motor noise affecting participants' performance in both tasks is equal. As the motor noise in the calibration task is not time-dependent, we assumed that all time-dependent performance improvement during the hidden target task reflected statistical learning, defined as the reduction in the uncertainty regarding the location of the hidden target.

\section{Participants successfully accumulate information and learn on a short time scale}

To establish that participants were in general able to learn on a short timescale, we initially focused on the first 10 trials of each hidden target block (Fig. 2B). In this case, in all 10 trials participants responded by using the same modality, either exclusively by pro-saccades, or 
A

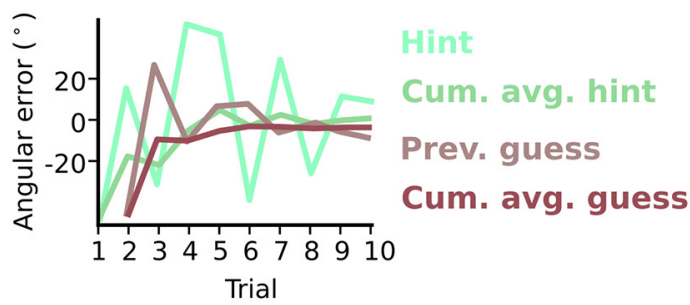

C

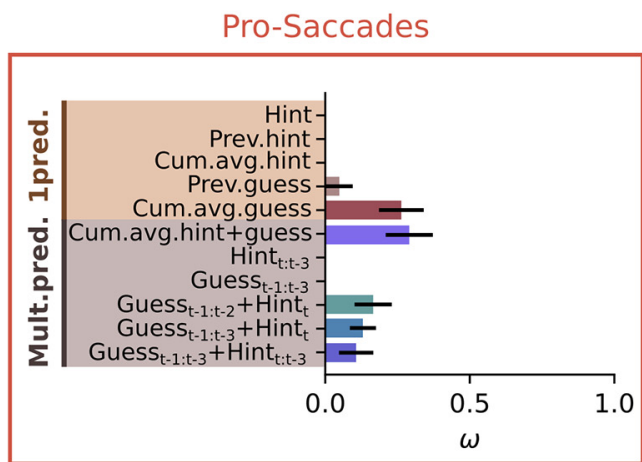

B

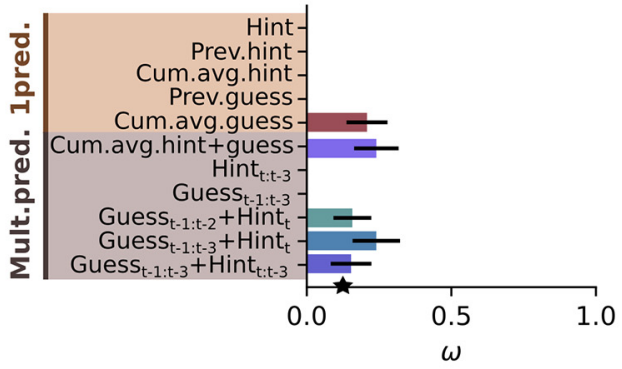

D

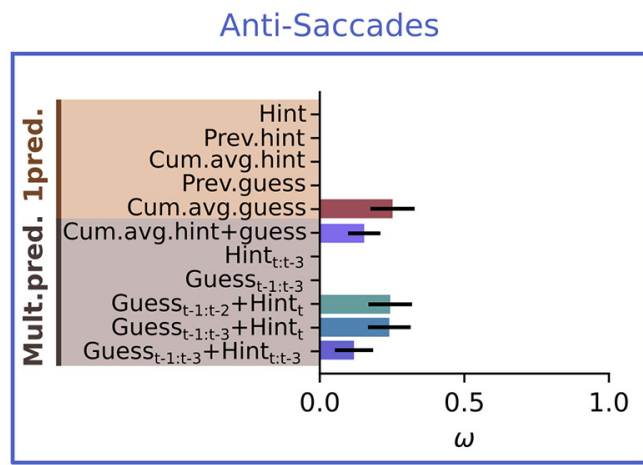

$\star\left(\right.$ Guess $_{\mathrm{t}-1: \mathrm{t}-3}+$ Hint $\left._{\mathrm{t}: \mathrm{t}-3}\right)$ :Modality

E

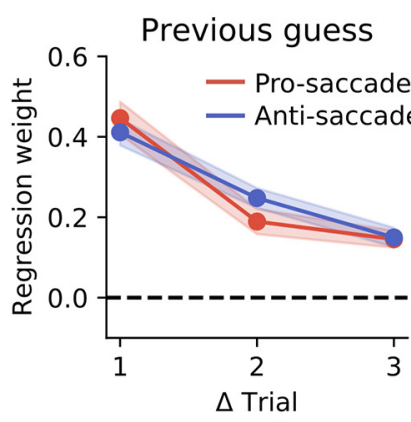

F

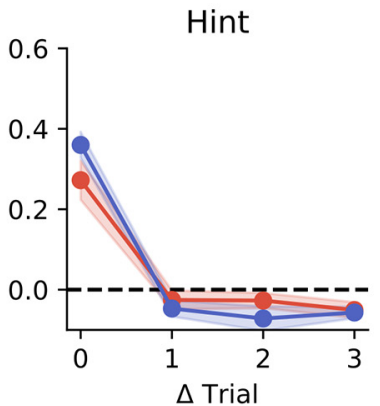

G

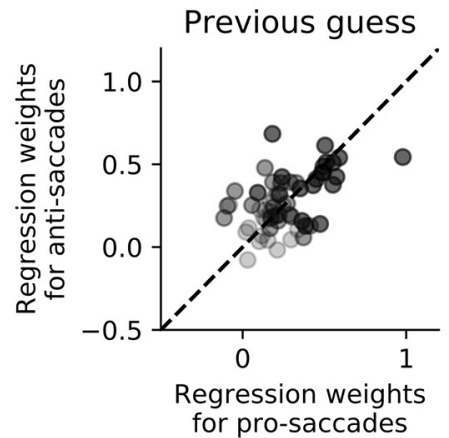

H

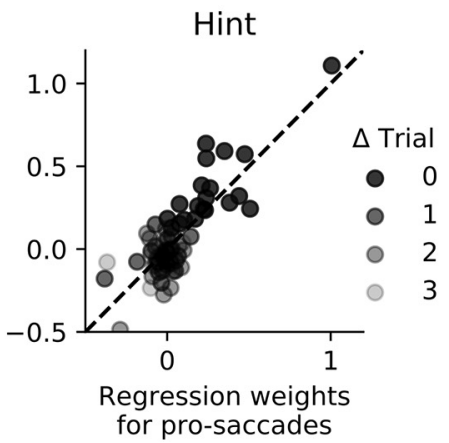

Figure 4. Learning strategy is similar across response modalities. A, Different predictors used to explain the participants' single trial estimates (i.e., angular error). $\boldsymbol{B}$, Model comparison between various single and multiple predictor models. Shown are the weights $\omega$, which represent the probability that a model is the best among the ones considered. Error bars indicate $\mathrm{SEM}(N=20)$. $\boldsymbol{C}, \boldsymbol{D}$, Same as $\boldsymbol{B}$ but performed on two different datasets, one consisting only of pro-saccade response trials $(\boldsymbol{C})$, the other consisting only of anti-saccade response trials $(\boldsymbol{D})$. $\boldsymbol{E}-\boldsymbol{H}$, Regression weights for a model including participants' last three guesses and the current and last three visual hints. Shaded area indicates SEM $(N=20)$. $\boldsymbol{E}$, Regression weights put on the last three guesses. $\boldsymbol{F}$, Regression weights put on the current, as well as the last three hints. $\boldsymbol{G}, \boldsymbol{H}$, Participants put similar weight on guesses and hints in pro-saccade and anti-saccade response trials (paired $t$ test for previous guess: $t=-0.38 p=0.70$; paired $t$ test for hint: $t=-0.26$ $p=0.79$ ). The weights put on previous hints and guesses were validated using separate models for hints and guesses (Extended Data Fig. 4-1). To select the relevant number of time steps in the past to include in the model comparison in B (see Table 1 for model definitions), we used a stepwise regression approach (Extended Data Fig. 4-2). Results shown in $\boldsymbol{B}$ were validated by varying the dataset used to fit the models (Extended Data Fig. 4-3), looking at best single subject models (Extended Data Fig. 4-4), and splitting the data according to task difficulty and response type (Extended Data Fig. 4-5).

exclusively by anti-saccades. To quantify performance, we calculated the absolute angular error between the saccade endpoint and the hidden target location (Fig. 2A). By comparing the average absolute angular error in the first five trials with the average absolute angular error in the last five trials, we found that most of the participants were able to improve their estimates of the target location (i.e., their guesses) during this short timescale (paired $t$ test: $t=7.25, p<0.0001, N=20$; Fig. $2 C$ ). In line with performance, participants' confidence about the accuracy of their guesses increased during the 10 trials (paired $t$ test: $t=$ $-4.39, p=0.0003, N=20$; Fig. 2D). Additionally, the absolute angular error of participants' guesses was lower than the absolute angular error of the visual hints, meaning that 

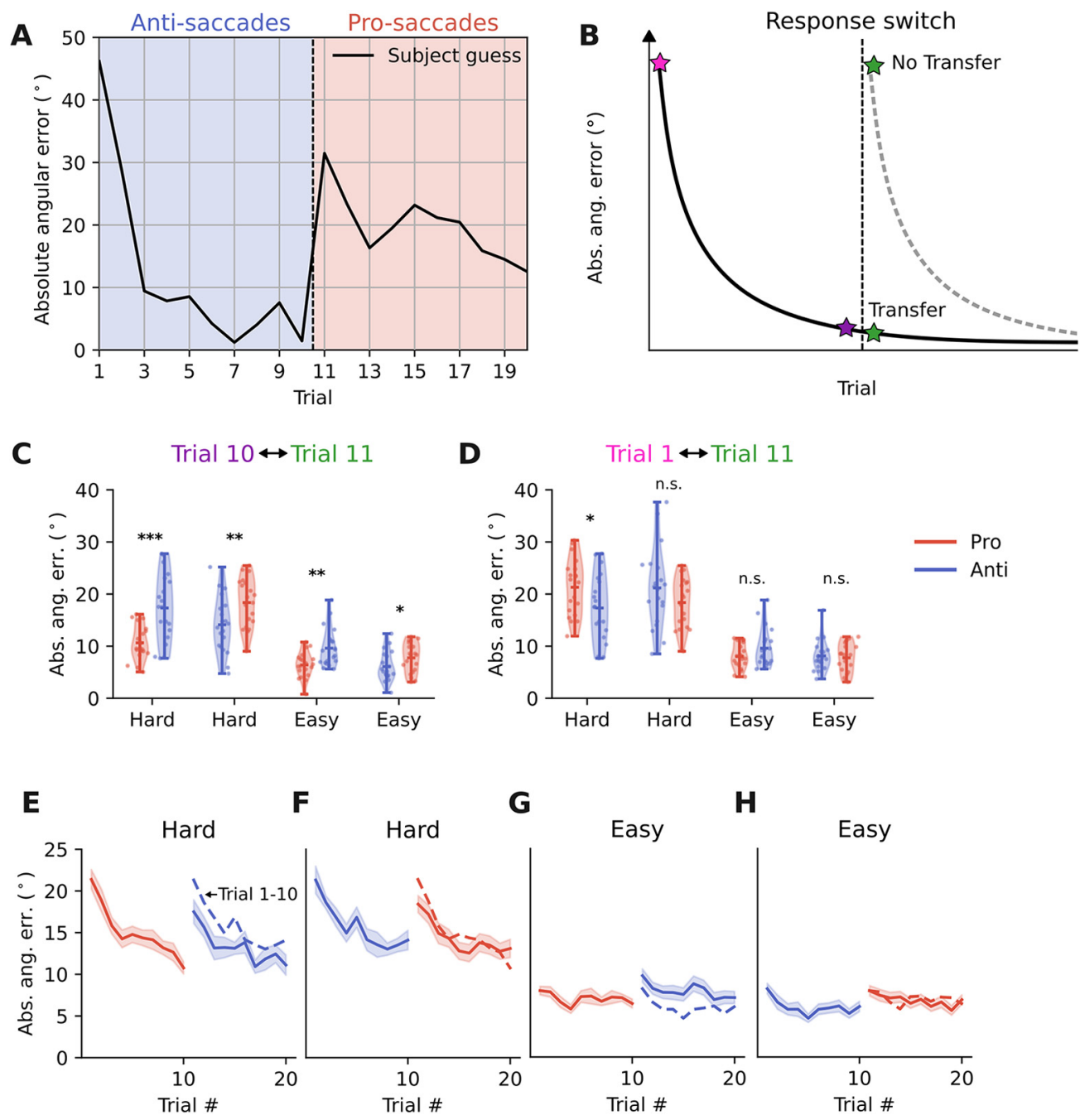

Figure 5. Drop in performance after response switch. $\boldsymbol{A}$, To test the knowledge transfer hypothesis, we analyzed all trials within a block, encompassing trials before and after the response switch. We specifically focused on the difference between trial 10 (before response switch) and trial 11 (after response switch). The same example block as in Figure 2 is shown. $\boldsymbol{B}$, If knowledge is transferred, we expect similar performance in trial 11 and trial 10. In contrast, if no knowledge is transferred, we expect similar performance in trial 11 and trial 1. To analyze the difference because of statistical learning only, we subtracted the motor error estimated from the calibration task to make pro-saccade and anti-saccade trials more comparable. $\mathbf{C}$, Comparison of performance in trial 10 and 11 for the four different experimental conditions (difficulty $\times$ pro/anti order). Each dot represents one subject and horizontal bars indicate mean and extreme values. $\boldsymbol{D}$, Same as $\boldsymbol{C}$ but for comparison between trial 1 and trial 11 ; ${ }^{* *} p<0.001,{ }^{* *} p<0.01,{ }^{*} p<0.05$, n.s. $\boldsymbol{p}>0.05$. $\boldsymbol{E}-\boldsymbol{H}$, Performance time course for different difficulty levels and pro-/anti-saccade orders. Dashed colored lines represent performance in trials 1-10. Shaded area indicates the SEM $(N=20)$. The results for performance were corroborated by analyzing confidence levels, which showed a similar drop from trial 10 to trial 11 (Extended Data Fig. 5-1, Fig. 5-2). We tested whether the drop in performance was related to the fact that subjects might have misunderstood the task. A control experiment (see Materials and Methods, experiment 2) confirmed that although subjects were aware that all 20 trials belonged to the same hidden target location, they were not able to integrate information across the response switch (Extended Data Fig. 5-3). Furthermore, we validated that the subtraction of the motor error is plausible and that there is no temporarily increased motor error after the response switch (Extended Data Fig. 5-4).

participants' guesses were closer to the center of the von Mises distribution compared with the presented visual hints (paired $t$ test: $t=4.92, p=0.0001, N=20$; Fig. $2 E$ ). This shows that participants were able to combine information across trials and thereby improve their estimates of the target location, rather than just following the current visual hint. Hence, we can conclude that participants showed some form of statistical learning during the first 10 trials of the hidden target task.

\section{Similarity of learning curves across response modalities}

To work out whether statistical learning is similar across response modalities, we contrasted the learning performance in pro-saccade versus anti-saccade trials in the hidden target task, as well as in the calibration task. First, we calculated the mean and the standard deviation of the respective angular error distributions, pooled across participants (Fig. $3 A, B$; Table 2), as well as the performance 

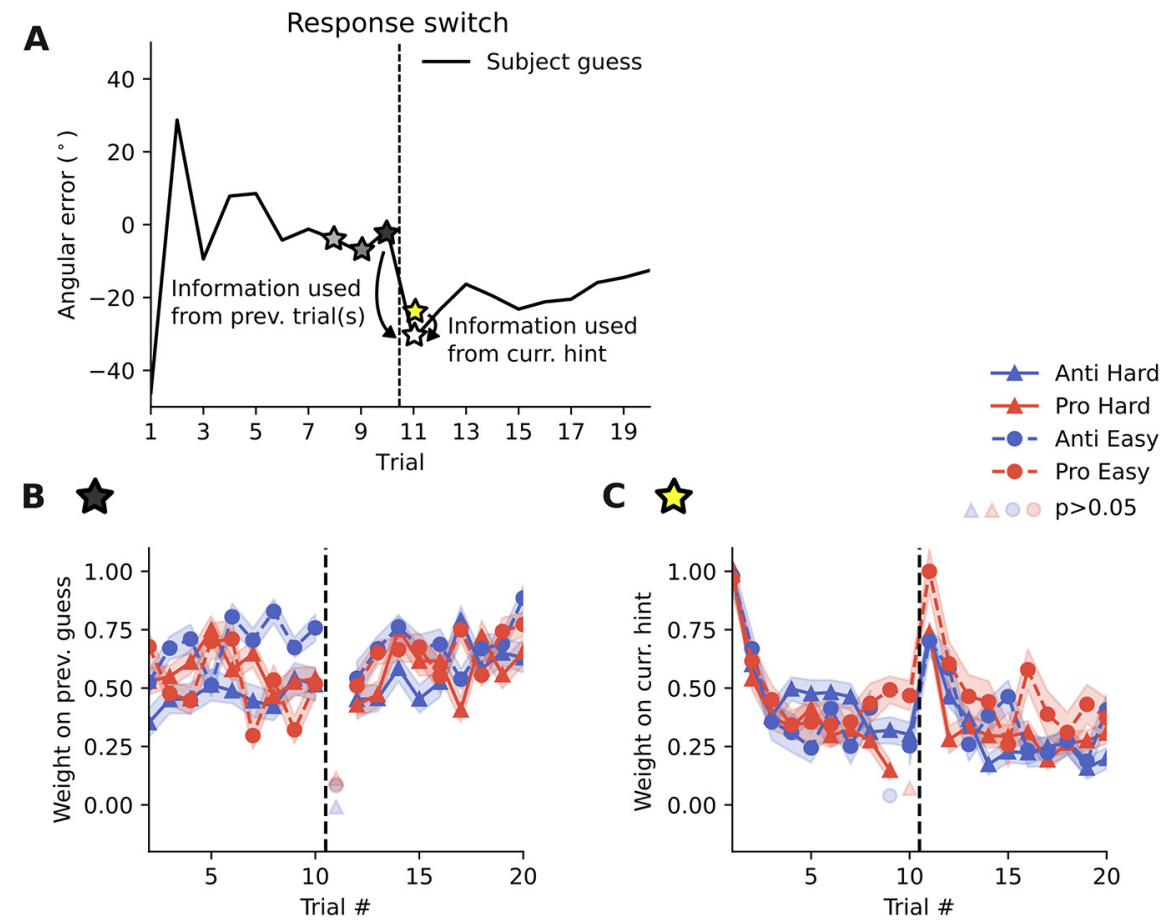

\section{D占}

E饮
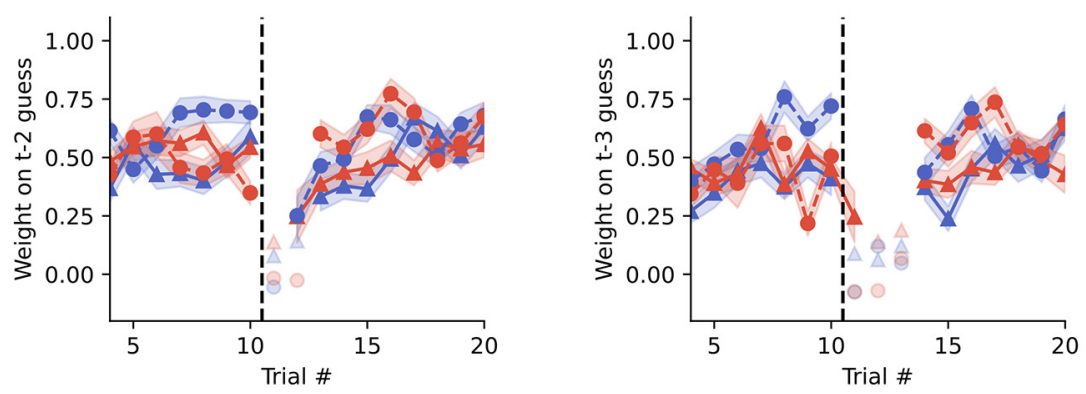

Figure 6 Almost no knowledge transfer between visuo-motor modalities. $\boldsymbol{A}$, To test whether there is any knowledge transferred from the experience with one response type to the other, we regressed the current guess against previous guesses/current hint. $\boldsymbol{B}$, Participants' estimates at trial 11 (after response switch) are independent of the estimates at trials 10 (before response switch). In contrast, at every other time point, participants use previous experience to inform their current guess. Shaded area here and in the remaining panels indicates the SEM $(N=20)$. $C$, At trial 11 , participants highly rely on the information coming from the current hint. $\boldsymbol{D}, \boldsymbol{E}$, Similar to the lack of transfer from one trial to the next $(\boldsymbol{B})$, there is also no transfer from trials further in the past across the response switch (trials 11-12 for t-2; trials 11-13 for t-3). In $\boldsymbol{B}, \boldsymbol{D}, \boldsymbol{E}$, non-significant regression weights with a $p>0.05$ are shown in opaque.

Table 1: Overview of the models used to assess sensorimotor statistical learning

\begin{tabular}{|c|c|c|}
\hline & Name & Equation \\
\hline$\overline{1}$ & Hint & Guess $_{t}=\beta_{0}+\beta_{1}$ Hint $_{t}$ \\
\hline 2 & Prev. hint & Guess $_{t}=\beta_{0}+\beta_{1}$ Hint $_{t-1}$ \\
\hline 3 & Cum. avg. hint & Guess $_{t}=\beta_{0}+\beta_{1}$ CumAvgHint $_{t}$ \\
\hline 4 & Prev. guess & Guess $_{t}=\beta_{0}+\beta_{1}$ Guess $_{t-1}$ \\
\hline 5 & Cum. avg. guess & Guess $_{t}=\beta_{0}+\beta_{1}$ CumAvgGuess $_{t}$ \\
\hline 6 & Cum. avg. hint + guess & Guess $_{t}=\beta_{0}+\beta_{1}$ CumAvgHint $_{t}+\beta_{2}$ CumAvgGuess $_{t}$ \\
\hline 7 & Hint $_{\mathrm{t}: \mathrm{t}-3}$ & Guess $_{t}=\beta_{0}+\beta_{1}$ Hint $_{t}+\beta_{2}$ Hint $_{t-1}+\beta_{3}$ Hint $_{t-2}+\beta_{4}$ Hint $_{t-3}$ \\
\hline 8 & Guess $_{t-1: t-3}$ & Guess $_{t}=\beta_{0}+\beta_{1}$ Guess $_{t-1}+\beta_{2}$ Guess $_{t-2}+\beta_{3}$ Guess $_{t-3}$ \\
\hline 9 & Guess $_{\mathrm{t}-1: \mathrm{t}-2}+$ Hint $_{\mathrm{t}}$ & Guess $_{t}=\beta_{0}+\beta_{1}$ Guess $_{t-1}+\beta_{2}$ Guess $_{t-2}+\beta_{3}$ Hint $_{t}$ \\
\hline 10 & Guess $_{\mathrm{t}-1: \mathrm{t}-3}+\mathrm{Hint}_{\mathrm{t}}$ & Guess $_{t}=\beta_{0}+\beta_{1}$ Guess $_{t-1}+\beta_{2}$ Guess $_{t-2}+\beta_{3}$ Guess $_{t-3}+\beta_{4}$ Hint $_{t}$ \\
\hline 11 & Guess $_{\mathrm{t}-1: \mathrm{t}-3}+$ Hint $_{\mathrm{t}: \mathrm{t}-3}$ & Guess $_{t}=\beta_{0}+\beta_{1}$ Guess $_{t-1}+\beta_{2}$ Guess $_{t-2}+\beta_{3}$ Guess $_{t-3}+\beta_{4}$ Hint $_{t}+\beta_{5}$ Hint $_{t-1}+\beta_{6}$ Hint $_{t-2}+\beta_{7}$ Hint $_{t-3}$ \\
\hline
\end{tabular}


Table 2: Angular error distribution for the calibration and the hidden target task

\begin{tabular}{|c|c|c|c|c|}
\hline Task & Modality & Mean & SD & Absolute angular error \\
\hline$\overline{\text { Calibration }}$ & Pro-saccade & $-0.1^{\circ}$ & $6.3^{\circ}$ & $3.5^{\circ}$ \\
\hline Calibration & Anti-saccade & $0.7^{\circ}$ & $9.5^{\circ}$ & $7.1^{\circ}$ \\
\hline Hidden target & Pro-saccade & $1.1^{\circ}$ & $16.9^{\circ}$ & $12.1^{\circ}$ \\
\hline Hidden target & Anti-saccade & $0.1^{\circ}$ & $18.4^{\circ}$ & $13.4^{\circ}$ \\
\hline Hidden target & Hints & $0.1^{\circ}$ & $20.6^{\circ}$ & $14.7^{\circ}$ \\
\hline
\end{tabular}

Mean and SD for distributions shown in Figure $3 A, B$. The rightmost column indicates the average absolute angular error in each task condition, estimated from the subjects' guess, or, for the last row, from the distribution of visual hints.

measure used here and in the following, the absolute angular error (Table 2).

For both modalities and both task difficulties, the absolute angular error in the hidden target task was higher than in the calibration task (pro hard: $t=13.8, p<0.0001$; pro easy: $t=8.8, p<0.0001$; anti hard: $t=14.1, p<$ 0.0001 ; anti easy: $t=2.9, p=0.0084$; paired $t$ test each with $N=20$ ). This makes sense as in the former task the target location was uncertain whereas in the latter it was not. Furthermore, the performance in pro-saccade and anti-saccade blocks was only significantly different during the calibration task, but not during the hidden target task (calibration task: $t=5.6, p<0.0001$; hidden target task easy: $t=1.7, p=0.1106$; hidden target task hard: $t=1.8$, $p=0.0903$; paired $t$ test each with $N=20$ ). To quantify this difference further, we calculated a modality difference index (described in Materials and Methods), which was consistently higher in the calibration task compared with the hidden target task (Fig. 3C). These results provide first evidence that statistical learning is similar for pro-saccade and anti-saccade response.

So far, we have shown that the average performance in the first 10 trials does not seem to depend on the modality used for indication, pro-saccades or anti-saccades. Nevertheless, the specific time course of statistical learning could still be modality dependent. For this, we next looked at the performance in a trial-dependent manner (Fig. 3D). As expected from the analysis in Figure 2C, we observed that the performance is slowly increasing with each trial, indicating that the participants were using the information given in each trial to improve their estimate of the hidden target location. This general behavior was also reflected in the time course of subjects' own confidence (Fig. $3 E$ ). In line with the previous, trial-independent analysis, we found that the performance for pro-saccades and anti-saccades was very similar (Fig. 3D, compare red and blue). This similarity seemed especially interesting when compared with the observed difference in motor error during the calibration task (Fig. 3D, dashed line). In summary, inspection of the learning curves of pro-saccade and anti-saccade indication supported our initial results suggesting that statistical learning is independent of the response modality.

\section{Performance is mostly suboptimal}

Since at each point in time, participants have only seen a limited number of samples from the underlying distribution of the target location (in form of visual hints), it is impossible to correctly estimate the distribution's mean, i.e., the hidden target location in our task. In principle, only for an infinite number of samples, the estimated mean equals the true distribution mean. For the hidden target task, this means that participants could only reach zero absolute angular error if an infinite number of trials were seen. Taking this into account, we can compare participants' performance to the time course of the theoretical statistical uncertainty because of seeing a limited number of samples (i.e., sampling error). This provides a lower bound $\gamma$ on participants' performance:

$$
\gamma=\sqrt{\gamma_{\text {Motor }}^{2}+\gamma_{\text {Hint }}^{2}}
$$

where $\gamma_{\text {Motor }}$ is the motor error measured in the calibration task and $\gamma_{\text {Hint }}$ is the absolute angular error related to seeing only a limited number of samples (the visual hints). Comparing participants' performance to this lower bound showed that for the first trial in each hidden target block, participants performed as well as they possibly can (Fig. 3F-J; Extended Data Fig. 5-4), independent of the task difficulty or the used response modality, pro-saccades or anti-saccades. The fact that the estimated lower bound matched subject performance in the first trial, where no learning has yet happened, also supports the plausibility of the assumption that the motor noise and the statistical noise can be added to derive an estimate of participants' performance (also see Extended Data Fig. 5-4). However, beyond the first trial and as soon as subjects needed to combine multiple samples to estimate the hidden target location, they performed suboptimally in most conditions, as can be seen in the difference of their estimates to the theoretical lower bound (Fig. 3F-J, black dashed line is optimal).

Interestingly, we found minor differences in terms of performance between the four different conditions we tested (pro/anti $\times$ hard/easy). Three out of four conditions showed clear suboptimal behavior (Fig. $3 F, H, J)$. However, in the condition where the visual hint distribution was more concentrated (i.e., the easy condition) and anti-saccades were used as the response modality, performance was close to optimal (Fig. 3G). As described before, this finding cannot be ascribed to the fact that our estimate of the motor error was flawed, thereby artificially improving the measured performance, as the estimate for Trial 1 closely matched what we observed in participants' behavior (Extended Data Fig. 5-4). We suspect that in this condition, performing an anti-saccade discourages a bias to follow the visually presented lines too much, and hence can be beneficial in producing optimal performance. Nevertheless, the differential learning of pro-saccade and anti-saccade responses with respect to an optimal lower bound on performance was not present in the hard task condition (Fig. $3 H-J$ ), indicating that the optimal learning for anti-saccades only occurred when the visual hints where narrowly distributed.

\section{Learning strategy is similar across response modalities}

So far, we have seen that statistical learning in the prosaccade and in the anti-saccade context is similar in 
terms of the absolute performance and the shape of the learning curve. Next, we wanted to test whether participants used different learning strategies in the pro-saccade and anti-saccade blocks. The first candidate strategy would be to only look at the visual hints in each trial, which would mean that no learning is happening, and that participants' behavior is only visually driven. Instead, the optimal strategy, which would result in the lower bound we calculated before, would be to calculate the cumulative average of every hint seen so far in each trial. Besides considering the hints, visually presented to the participants, we can also imagine that the behavior is driven by an internal state that promotes looking close to where one has been looking before, i.e., to follow previous guesses (Fig. 4A). To test these different hypotheses, we fit several single predictor linear regression models corresponding to each strategy (see Table 1). Through a model comparison (see Materials and Methods), we found that the best single predictor model is the cumulative average of previous guesses (Fig. 4B; Extended Data Figs. 4-3, 4-4). Splitting the data into pro-saccade and anti-saccade blocks and repeating the analysis showed that the best single predictor model does not depend on the used response type (Fig. 4C,D). Thus, this provides another piece of evidence that statistical learning in the presented task is independent of the response type.

In a second step, we tested several multipredictor models to get a more detailed view on participants' learning strategy. To determine the number of trials in the past potentially having an influence on the current decision, we conducted a stepwise linear regression analysis, where all previous guesses and all presented hints thus far were included to estimate participant's angular error in trial 10 (Extended Data Fig. 4-2). This showed that for most participants, models with a low number of predictors performed as well as or better than models including all past trials (Extended Data Fig. 4-2A-C). Furthermore, we found that the predictors most often included in the best model were the current visual hint and the three previous guesses (Extended Data Fig. 4-2D). Based on these results, we chose to include information from up to three timesteps in the past and specifically compared five different options of how previous guesses and presented visual hints can be combined (Table 1). The model comparison revealed that multipredictor models which relied on combinations of external and internal information, i.e., visual hints and previous guesses, performed much better than models that were based on only one of the two sources of information (i.e., either the previous three guesses or the current and previous three visual hints; Fig. 4B). This was consistent across subjects (Extended Data Fig. 4-4). Again, splitting the data into pro-saccade and antisaccade blocks did not affect the main trend (Fig. 4C,D), nor did splitting the data into hard and easy task difficulty (Extended Data Fig. 4-5).

Lastly, to find the exact weighting participants put on their previous guesses and the current and previous hints, depending on the used response type, we fitted a model with all three previous guesses, the current and three previous hints, and the response type as predictors. We found that participants used external information only from the current trial, ignoring the hints from previous trials (Fig. 4F). Instead, to combine information across trials they relied on their internal estimations from the past (Fig. $4 E$ ). We obtained similar results when models that only included either the past guesses or the past visual hints were tested (Extended Data Fig. 4-1), excluding the possibility that our results were biased by the inherent covariation of previous guesses and previous visual hints. Including the response type in the model allowed to estimate separate regression weights for pro-saccades and anti-saccades. Again, we did not find any significant difference in the weighting participants put on their own guesses versus given hints, depending on whether they use pro-saccades or antisaccades for response (Fig. $4 E-H$; paired $t$ test for previous guess: $t=-0.38 p=0.70$; paired $t$ test for hint: $t=-0.26$ $p=0.79$ ). In summary, we found that participants used a very similar learning strategy to solve the task, regardless of the response type.

\section{Drop in performance after response switch}

Despite the similarity in learning performance and strategy, hinting at a general algorithm used for statistical learning in our task, it is still possible that learning happens for each visuo-motor modality in a very specific manner and that both just look similar in terms of performance and strategy. In this case, it would not be possible to generalize between modalities. To test this, we included trial 11 until trial 20 where participants had to continue looking for the same hidden target (and were also instructed that all 20 trials belong to one hidden target), but had to use the other visuo-motor modality than in trial 1 until trial 10 (Fig. 5A). We considered two alternative hypotheses. The first states that participants learn in a modality-independent fashion and store the acquired knowledge in an abstract form. This would allow for a complete transfer of previous experience to the new response modality (Fig. 5B, black solid line). The second hypothesis would be that participants perform trial 11 until trial 20 as if there was no previous experience, which would suggest a response modality-specific representation of the learned knowledge (Fig. $5 B$, gray dashed line). To test which of the two hypotheses better matched the data, we compared the performance in trial 10 (last trial before response switch) to the performance in trial 11 (first trial after response switch; Fig. 5C). Since trials before and after the response switch were performed using different response modalities and since pro-saccades and anti-saccades are associated with different motor errors (Fig. 2), we subtracted the respective motor error estimated from the calibration task from participants' absolute angular errors during the hidden target task. This procedure was validated by demonstrating that the resulting estimate closely matched the ground truth statistical error, which in trial 1 is simply the uncertainty related to the spread of the visual hint distribution (Extended Data Fig. 5-4C-F). A repeated measures three-way ANOVA 
was performed on participants' absolute angular error as the dependent factor and task difficulty, trial number (10 or 11), and pro-/anti-saccade order as independent factors. We found significant main effects of difficulty $(p<0.0001)$ and trial number $(p<0.0001)$, but the main effect of pro-/anti-saccade order was not significant $(p=0.291)$. There were also significant interaction effects between pro-/anti-saccade order and difficulty $(p=0.004)$, as well as between trial number and difficulty $(p=0.006)$. Post hoc paired $t$ tests showed that the performance in trial 11 was overall worse than in trial 10 , with a significant drop in performance in all four conditions (trial 11-trial 10 for pro-saccade first/hard: $t=4.05, p=0.0007$; for anti-saccade first/ hard: $t=3.17, p=0.005$; for pro-saccade first/easy: $t=3.01$, $p=0.007$; for anti-saccade first/easy: $t=2.20, p=0.04$; compare Fig. $5 C, E-H)$. This showed that there is an overall drop in performance after the response switch suggesting that the knowledge acquired during the first 10 trials is not fully transferred to trials after a response switch. The drop in performance is not likely to be because of a temporarily increased motor error after a switch in response type, as we did not observe a similar pattern during the calibration task (paired $t$ test to compare absolute angular error in trial 20 and trial 11 of the calibration task: $t=-1.5, p=0.15$; Extended Data Fig. 5-4A). These results were corroborated by analyzing confidence ratings, as we also observed a drop in confidence at trial 11 compared with trial 10, in line with our results on performance (paired $t$ test trial 11-trial 10, $t=3.7, p=0.0016$; Extended Data Figs. 5-1, 5-2). Together, these results suggest that although the learning curves and strategies for pro-saccade and anti-saccade responses were highly similar (Figs. 3, 4), there was no full knowledge transfer from one response type to the other.

To test whether there is at least a minor improvement in performance related to experiencing 10 trials of the opposite response type, we compared the performance in trial 11 (after the response switch) with the naive performance in trial 1 (Fig. 5D). We used the same ANOVA analysis as before, only replacing trial 10 with trial 1 . There was a statistically significant effect of difficulty $(p<0.0001)$, but not trial number $(p=0.103)$, or pro-/anti-saccade order $(p=0.789)$. There also was a significant interaction effect between difficulty and trial number $(p=0.003)$. Post hoc paired $t$ tests showed that there was a significant improvement in performance from trial 1 to trial 11 only in the hard task condition when pro-saccade response was followed by anti-saccade response (trial 11- trial $1, t=$ $-2.28, p=0.034$ ), but not in any other of the remaining three conditions (hard/anti-first: $p=0.207$; easy/pro-first: $p=0.168$; easy/anti-first: $p=0.679$ ). Together, these results suggest that there is only very limited knowledge transfer from one response condition to the other, as performance was slightly increased compared with naive levels only in one out of four conditions (hard/pro-first).

As comparing performance before and after the modality switch necessarily includes the factor that pro-saccades and anti-saccades have inherently different performance because of motor noise (compare Fig. $2 A, B$ ), we also compared the performance within the same modality, either pro-saccade or anti-saccade response, between trial 1 and trial 11. To analyze whether performance overall improved in trial 11 , compared with 1 , we performed a similar repeated measures three-way ANOVA with modality, difficulty, and trial number as independent factors. Here, we did not subtract the motor error, since the effect of trial number was compared within, and not across response modalities, as previously done. We found a significant main effect of difficulty $(p<0.0001)$, but not modality or trial number, similar to our previous analysis subtracting the motor error. Additionally, we found a significant interaction effect between difficulty and trial number $(p=0.0020)$. Post hoc $t$ tests confirmed that in only one out of the four experimental conditions there was a statistically significant improvement between trial 1 and trial 11 (trial 11 - trial 10 hard/pro: $t=-2.2, p=0.041$; hard/anti: $t=-1.75$, $p=0.097$; easy/pro: $t=-0.2, p=0.837$; easy/anti: $t=1.9$, $p=0.072$ ). In summary, this analysis confirmed the results we obtained when comparing pro-saccades and anti-saccades directly, thus ruling out the possibility that our results are merely an artifact of subtracting the motor error measured in the calibration task.

\section{Almost no knowledge transfer between visuo-motor modalities}

As modeling showed that participants relied on their previous guesses rather than the previously seen visual hints (Fig. 4E,F), we wished to test whether this is also the case across the time that a response switch occurs. For this, we calculated the regression weights on previous guesses in a time-dependent manner (Fig. 6A). If knowledge is not transferred between different visuo-motor modalities we would predict: (1) participants highly rely on the visual hint in trial 11 and do not use previous guesses to inform their decision; (2) they are also not able to use previous guesses further in the past, if these guesses were obtained before the response switch. Our analysis confirmed these two predictions. We found that participants did not use their previous guess from trial 10 (before the response switch) to inform their estimate in trial 11 (after the response switch; Fig. 6B). In contrast, at all other time points they used previous guesses to inform their current decision. As participants did not use their previous experience in trial 11, we expected that they instead highly relied on the hint presented in trial 11 . Regression analysis confirmed this hypothesis showing a peak at trial 11, similar to the peak at trial 1 (Fig. 6C). Furthermore, inspired by the initial modeling results on participants' strategies (Fig. 4), we tested the influence of previous guesses further in the past [two trials (Fig. 4D) and three trials (Fig. 4E)]. Again, we found that there is mostly no influence across the response switch. In summary, these analyses showed that participants' behavior after a response switch was not guided by previous experience, obtained in the opposite response condition, thus suggesting a lack of full knowledge transfer across conditions.

One possible explanation for these results is that participants had difficulties in understanding that the hidden target location had remained the same across first and second 10 trials (i.e., between trial 10 and trial 11). To rule out this possibility, we performed a second experiment 
with an independent set of participants $(N=20)$ where we further emphasized that the target location was the same across all 20 trials (for details, see Materials and Methods). Furthermore, we asked participants at the end of each block whether they were aware that all 20 trials performed so far belonged to the same hidden target. We obtained overall similar results in this experiment (Extended Data Fig. 5-3), as learning performance, as well as information transfer and confidence were disturbed after the response switch between trial 10 and trial 11 , although participants were explicitly instructed that all 20 trials belonged to the same hidden target and were also aware of this (Extended Data Fig. 5-3B). This ruled out the possibility that the inability to transfer knowledge between response modalities is because of a cognitive misunderstanding of the task.

\section{Discussion}

The aim of the current study was to characterize the dynamics of prior learning and its dependence on the type of motor response used to report choices. We found that participants could learn a sensorimotor prior within a few trials, with the learning time course being mostly independent of the response type (pro-saccades or anti-saccades). By using a model-comparison approach, we further demonstrated that participants relied more on their own guesses from previous trials compared with visual hints provided in previous trials, again, independent of the response type. This suggests that prior knowledge is represented in terms of previous motor actions and not incoming, external information provided by the visual hints. To verify this hypothesis, we tested whether participants could generalize their learned prior knowledge from one motor context to the other, a switch from pro-saccades to anti-saccades or vice versa. We found that switching the response type caused a drop in performance, close to resetting to naive levels, indicating that experience from one response type could not be fully transferred to the other. This was the case even despite explicit instructions and participants' awareness that pro-saccade and antisaccade trials belonged to the same hidden target location. Our results suggest that humans learn sensorimotor priors through monitoring their previous motor decisions rather than external sensory hints. The dependence of learning on past motor decisions discourages generalization of the learned knowledge to conditions where a different visuo-motor mapping is needed.

Our findings suggest that prior knowledge is represented in a motor specific manner during early learning, which is in line with previous studies reporting motor specific priors in different paradigms (Roach et al., 2017; Chambers et al., 2019). We could furthermore identify one potential reason for why generalization is not possible in such contexts, as we found that participants do not memorize the external information from previous trials (visual hints in our case), but instead they memorize their own actions in each trial (Fig. 4E,F). As our task required an estimation in every trial, indicated by either a pro-saccade or an anti-saccade, the memory of each trial's decision was probably represented as the motor action taken to indicate the guess. This could also explain why tasks which do not require an explicit response via a motor action are more generalizable (Roach et al., 2017). In these cases, the memory from previous trials is potentially formed in a more abstract way, as participants only have to "think" about their decision, but not perform any specific action. The finding that prior knowledge is built on internal decisions, compared with external cues, could therefore unify some previous controversial findings about prior generalization.

Despite the suggested motor-specific formation of prior knowledge, the algorithm to combine previous experiences to inform the current decision seemed to be similar for both tested response contexts (Fig. 4E-H). This suggests that there is a general procedure for how humans combine previous experiences. However, whether prior knowledge can generalize or not depends on the specific manner through which previous experience or decisions are stored in memory (e.g., in terms of motor actions or more abstract decisions). In other words, although at an algorithmic level learning is independent of the response modality, the learned information is stored with a format that is specific for each modality. This explanation is in line with the previously proposed dissociation between learning a policy versus knowledge (Chambers et al., 2019), and further narrows the space of testable predictions regarding the neural implementation of these different types of learning, as the well-described neuronal machinery of pro-saccades and anti-saccades (Munoz and Everling, 2004; Ford et al., 2005; Juan et al., 2008) could allow a characterization of how the two types of learning occur in the brain, for instance through using neuroimaging techniques.

Our study is different from previous studies as it does not test prior learning in a condition where there is also sensory uncertainty (Berniker et al., 2010; Dekleva et al., 2016). In these task designs, participants are asked to perform a task trial-by-trial and are not explicitly told to combine information across trials. Prior learning in these cases is therefore implicit and potentially unconscious. Furthermore, learning is mostly observed by analyzing how participants combine the noisy sensory information in a given trial with the formed prior. It is therefore not directly possible to resolve which of the two is learned, as observed changes in this combination could potentially come from a changed likelihood distribution, from a changed prior distribution, or from changes in both distributions. Because of these limitations, we designed our task such that the sensory information in each trial is given by a clearly visible hint. We then explicitly asked the participants to combine the information across trials to find the hidden target location. Compared with previous prior learning studies (Berniker et al., 2010), we could therefore directly examine the ability to learn statistical regularities over trials. This more general statistical learning context has also been studied previously, for example showing that learning can happen rapidly within a dozen trials if feedback is provided (Chukoskie et al., 2013). However, to our knowledge, pure statistical learning, without trial-by-trial feedback, together with generalization has not been studied in this context. 
The two different task contexts we investigated in this work are distinct to previous studies, as we did not test generalization from one effector to another, such as performing a task with the right hand and switching to the left (Hewitson et al., 2018; Kumar et al., 2020), or switching from a motor to a perceptual task (Chambers et al., 2019). Instead, our two contexts represent two distinct cue-action mappings, though performed with the same motor system (oculomotor). By cue-action mapping we mean that participants had to indicate their guess (the internal cue) with two different response types, pro-saccades and anti-saccades (the actions), dependent on the task context. Potentially, generalization could be easier between different cue-action mappings compared with generalization between different effectors or tasks. In fact, previous studies have shown that probabilistic manipulations employed in either pro-saccades or anti-saccades affect both response types, suggesting that some form of statistical information is transferred across the two response modalities (Liu et al., 2010; Chiau et al., 2011; Pierce et al., 2015), albeit these studies have employed a task where statistical uncertainty is relatively low and does not have to be actively learned. Given the specific design of our task, we cannot differentiate whether participants form a motor independent spatial prior, which is aligned to the given cue-action mapping, or whether they form their prior directly at the motor level. In both cases generalization would fail, matching our experimental observation. Potentially, participants learn a spatial representation in the pro-saccade context, where the correct estimate lies close to their performed motor action endpoint. Then, in the anti-saccade context, they do not follow this estimate and solely invert their motor plan, but instead they form a "pro" representation of the hidden target location in this new context, where again the performed motor action endpoint is close to the acquired spatial estimate. In this interpretation, anti-saccades are not real anti-saccades, but pro-saccades relative to the participants' estimate and only visual information is inverted. What speaks for this interpretation is the fact that participants seemed to be closer to the optimal learning performance in the anti-saccade condition (Fig. 3F,G), although this was only the case when the visual hints were narrowly distributed. Interestingly, the only instances where some degree of generalization across response contexts was observed (i.e., when performance after response switch was better than naive levels) occurred when participants performed the hard task condition. Since higher uncertainty in our task discourages a close mapping between motor actions and visual cues (participants learn that the actual cue location is not necessarily near the visual hint), it may instead enable a more context-independent integration of information and formation of abstract knowledge, thereby promoting generalization. However, this abstraction seemed to be incomplete as performance was still affected by a change in response context.

Previous studies examining the effects of statistical regularities in the oculomotor system have primarily focused on how learned statistical information influences saccadic parameters, whereas the involvement of saccades during the learning process itself has been largely ignored. One line of research has investigated how the probabilistic information related to the frequency of appearance of the target in one out of a fixed set of possible locations affects saccadic parameters, both for pro-saccades (Carpenter and Williams, 1995) and anti-saccades (Koval et al., 2004; Liu et al., 2010). These studies have reported faster and more accurate gaze shifts toward high- compared with low-probability locations, and neurophysiological recordings have shown that these behavioral effects are because of an enhanced buildup of neuronal activity preceding saccades toward high-probability locations, observed in the superior colliculus (SC) and frontal eye fields (FEFs; Basso and Wurtz, 1997; Dorris and Munoz, 1998; Everling and Munoz, 2000; for evidence from human neuroimaging studies, see also Liu et al., 2011). Another line of research has investigated how the relative probabilities of pro-saccade versus anti-saccade response types affect saccadic parameters, showing for instance that increasing the relative probability of antisaccades versus pro-saccades may eliminate the typical anti-saccades' cost (Chiau et al., 2011; Pierce et al., 2015). The latter probabilistic effects were shown to be associated with modulations in cognitive control regions of the brain (Pierce and McDowell, 2016). Both location and response type probabilistic effects have been reported in settings where statistical uncertainty is relatively low and does not have to be actively learned. One novel aspect of the current study is to test how spatial priors can be learned through eye movements under more complex settings where the possible target locations are not fixed, and probabilities could only be inferred through tracking information over time, which to the best of our knowledge has not been investigated before. Furthermore, pro-saccades and anti-saccades in our study were not tested in terms of how they are affected by the end result of statistical learning, but were examined with respect to how they are involved in active accrual of statistical information from the environment. Despite these differences, our findings are overall in line with the general conceptual framework put forward by previous studies, namely that statistical information is faithfully encoded in the oculomotor system. Future studies will be needed to elucidate where in the oculomotor system neural responses exhibit a similar learning dynamic compared with the effects we observed here. We can, however, speculate that our effects are likely to be reflected in the same areas where location probability effects have been previously reported, namely SC and FEF, although the pattern of learning-related neural modulation might differ from a simple change in neuronal preparatory responses. Our findings also inspire future neuroimaging studies that are shown to be well-suited for differentiation of brain networks underlying pro-saccades versus anti-saccades (Munoz and Everling, 2004; Ford et al., 2005; Juan et al., 2008) and can be extended to contrast brain activations when statistical learning occurs through pro-saccades versus anti-saccades. This will allow to elucidate whether a learned statistical prior in one response modality is locally stored in networks specialized for that modality, as our findings suggest, or is represented by a distributed code encompassing brain areas that underlie both saccadic response types.

Our setup allowed us to simultaneously evaluate participants' performance, as well as their confidence in their 
given estimation. Interestingly, confidence also decreased after the response type switch, suggesting that participants were aware that they cannot generalize between both contexts. On the other hand, a control experiment, where we explicitly asked participants to indicate after each block whether they were aware that both response type contexts belonged to the same hidden target estimation, showed that that they knew that information could be combined across both contexts (Extended Data Fig. 5-3B). Together, these results suggest that participants' inability to transfer knowledge from one response context to the other was not because of conscious misunderstanding, but more likely because of the specific mechanisms of how the prior is formed unconsciously.

Although our results, especially the inability to generalize across different motor contexts, suggest that statistical learning is implemented in a low-level, motor-dependent way (Figs. 5, 6), there might be potentially alternative explanations for why we could not see generalization in our experiment. In the following we will discuss these alternative interpretations. One potential explanation for the lack of generalization could be an interference between the internal memory of the target location, which is disturbed by the information slide, presented between trial 10 and trial 11, indicating response switch. Another possibility is that participants would need to train on our task for more than one session, such that they can learn to adapt their way of forming the prior knowledge to something which allows for generalization across response contexts. Furthermore, in our experiments learning blocks were short and participants learned the prior distribution of the target within only 10 trials. To the best of our knowledge, the speed with which statistical regularities can be learned and its relation to the generalization across contexts has not been extensively investigated in the past. However, a few elegant studies have shown that statistical learning can occur within a similar timescale as investigated here. For instance, Turk-Browne et al. (2009) showed that learning-related changes in brain activity start to emerge after the first few repetitions of visual statistical regularities. It has also been shown that the mean compared with the variance of prior distributions can be learned rapidly, within only a few trials (Berniker et al., 2010). Based on these previous results and our current findings, we predict that some properties of the statistical priors can be learned very rapidly in most environments. However, it is possible that the short timescale of only 10 trials is not enough to form an abstract representation of the estimated target location that can be generalized across different contexts. Potentially, initial learning is motor-dependent, but over time this is transformed to a motor-independent knowledge, which can then be transferred to other motor contexts. We also note that in our experiment, switches in response modality were fully predictable as they always occurred after the first 10 trials of each block. This design was employed to reduce participants' uncertainty regarding the required response, as predictable task switches are in general associated with lower switch costs (Monsell, 2003), and in the specific context of pro-saccades and anti-saccades it has been shown that switch costs between response types is minimal when response types are predictable, i.e., in blocked compared with interleaved designs (Zeligman and Zivotofsky, 2017). It is therefore possible that environments in which changes in response context occur unpredictably or have to be inferred from past information (Sherman and Turk-Browne, 2020) will warrant a more protracted learning of statistical information and higher switch costs. Finally, one major reason for a motor-context dependent learning could be that we did not provide external feedback to guide the learning process, as done in some of the previous studies (Chukoskie et al., 2013). Instead, participants had to rely solely on their internal feedback, potentially coming from the motor system. Future studies will be needed to test these possible explanations.

Spatially directed movements such as saccadic eye movements and reaching are an integral part of our daily activities and acquired skills (e.g., imagine a cellist or a tennis player). Both types of movements are profoundly influenced by statistical priors (Körding and Wolpert, 2004; Brodersen et al., 2008). Furthermore, saccadic eye movements provide detailed sensory information about a scene and are tightly linked to the allocation of attention, hence being instrumental for active vision (Munoz, 2002; Wurtz, 2015). In comparison to the saccadic eye movement, reaching movements have enjoyed a more rigorous characterization of the learning dynamics (Berniker et al., 2010; Fernandes et al., 2014; Chambers et al., 2019; Yin et al., 2019). Inspired by these studies, we characterized the dynamics of statistical learning through eye movements, that are a more accessible motor plan to be tested in the laboratory, and the learned information acquired through their execution can directly impact the very way that the brain samples sensory information (Parr and Friston, 2017).

\section{References}

Basso MAA, Wurtz RHH (1997) Modulation of neuronal activity by target uncertainty. Nature 389:66-69.

Battaglia PW, Hamrick JB, Tenenbaum JB (2013) Simulation as an engine of physical scene understanding. Proc Natl Acad Sci USA 110:18327-18332.

Berniker M, Voss M, Kording K (2010) Learning priors for Bayesian computations in the nervous system. PLoS One 5:e12686.

Brainard DH (1997) The psychophysics toolbox. Spat Vis 10:433436.

Brodersen KH, Penny WD, Harrison LM, Daunizeau J, Ruff CC, Duzel E, Friston KJ, Stephan KE (2008) Integrated Bayesian models of learning and decision making for saccadic eye movements. Neural Netw 21:1247-1260.

Burnham KP, Anderson DR (2004) Multimodel inference. Sociol Methods Res 33:261-304.

Carpenter RH, Williams MLL (1995) Neural computation of log likelihood in control of saccadic eye movements. Nature 377:59-62.

Chambers C, Fernandes H, Kording KP (2019) Policies or knowledge: priors differ between a perceptual and sensorimotor task. J Neurophysiol 121:2267-2275.

Chiau HYY, Tseng P, Su JHH, Tzeng OJLL, Hung DL, Muggleton NG, Juan CHH (2011) Trial type probability modulates the cost of antisaccades. J Neurophysiol 106:515-526.

Chukoskie L, Snider J, Mozer MC, Krauzlis RJ, Sejnowski TJ (2013) Learning where to look for a hidden target. Proc Natl Acad Sci USA 110 [Suppl 2]:10438-10445. 
Cornelissen FW, Peters EM, Palmer J (2002) The eyelink toolbox: eye tracking with MATLAB and the psychophysics toolbox. Behav Res Methods Instrum Comput 34:613-617.

Dekleva BM, Ramkumar P, Wanda PA, Kording KP, Miller LE (2016) Uncertainty leads to persistent effects on reach representations in dorsal premotor cortex. Elife 5:e14316.

Dorris MCC, Munoz DPP (1998) Saccadic probability influences motor preparation signals and time to saccadic initiation. $J$ Neurosci 18:7015-7026.

Ernst MO, Banks MS (2002) Humans integrate visual and haptic information in a statistically optimal fashion. Nature 415:429-433.

Everling S, Munoz DPP (2000) Neuronal correlates for preparatory set associated with pro-saccades and anti-saccades in the primate frontal eye field. J Neurosci 20:387-400.

Fernandes HL, Stevenson IH, Vilares I, Kording KP (2014) The generalization of prior uncertainty during reaching. $J$ Neurosci 34:11470-11484.

Ford KA, Goltz HC, Brown MRG, Everling S (2005) Neural processes associated with antisaccade task performance investigated with event-related fMRI. J Neurophysiol 94:429-440.

Frost R, Armstrong BC, Siegelman N, Christiansen MH (2015) Domain generality versus modality specificity: the paradox of statistical learning. Trends Cogn Sci 19:117-125.

Hewitson CL, Sowman PF, Kaplan DM (2018) Interlimb generalization of learned bayesian visuomotor prior occurs in extrinsic coordinates. eNeuro 5:ENEURO.0183-18.2018.

Juan $\mathrm{CH}$, Muggleton NG, Tzeng OJL, Hung DL, Cowey A, Walsh V (2008) Segregation of visual selection and saccades in human frontal eye fields. Cereb Cortex 18:2410-2415.

Kiryakova RK, Aston S, Beierholm UR, Nardini M (2020) Bayesian transfer in a complex spatial localization task. J Vis 20:17-18.

Körding KP, Wolpert DM (2004) Bayesian integration in sensorimotor learning. Nature 427:244-247.

Koval MJ, Ford KA, Everling S (2004) Effect of stimulus probability on anti-saccade error rates. Exp Brain Res 159.2:268-272.

Kumar A, Panthi G, Divakar R, Mutha PK (2020) Mechanistic determinants of effector-independent motor memory encoding. Proc Natl Acad Sci USA 117:17338-17347.

Liu CL, Chiau HY, Tseng P, Hung DL, Tzeng OJL, Muggleton NG, Juan $\mathrm{CH}$ (2010) Antisaccade cost is modulated by contextual experience of location probability. J Neurophysiol 103:1438-1447.

Liu CL, Tseng P, Chiau HY, Liang WK, Hung DL, Tzeng OJL, Muggleton NG, Juan CH (2011) The location probability effects of saccade reaction times are modulated in the frontal eye fields but not in the supplementary eye field. Cereb Cortex 21:1416-1425.
Manohar SG (2019) Matlib: MATLAB tools for plotting, data analysis, eye tracking and experiment design (Public). https://doi.org/ 10.17605/OSF.IO/VMABG.

Monsell S (2003) Task switching. Trends Cogn Sci 7:134-140.

Munoz DP (2002) Commentary: saccadic eye movements: overview of neural circuitry. Prog Brain Res 140:89-96.

Munoz DP, Everling S (2004) Look away: the anti-saccade task and the voluntary control of eye movement. Nat Rev Neurosci 5:218228.

Parr T, Friston KJ (2017) The active construction of the visual world. Neuropsychologia 104:92-101.

Pierce JE, McDowell JE (2016) Modulation of cognitive control levels via manipulation of saccade trial-type probability assessed with event-related BOLD fMRI. J Neurophysiol 115:763-772.

Pierce JE, McCardel JB, McDowell JE (2015) Trial-type probability and task-switching effects on behavioral response characteristics in a mixed saccade task. Exp Brain Res 233:959-969.

Roach NW, McGraw PV, Whitaker DJ, Heron J (2017) Generalization of prior information for rapid Bayesian time estimation. Proc Natl Acad Sci USA 114:412-417.

Sato Y, Kording KP (2014) How much to trust the senses: likelihood learning. J Vis 14:13-13.

Sherman BE, Turk-Browne NB (2020) Statistical prediction of the future impairs episodic encoding of the present. Proc Natl Acad Sci USA 117:22760-22770.

Tenenbaum JB, Griffiths TL, Kemp C (2006) Theory-based Bayesian models of inductive learning and reasoning. Trends Cogn Sci 10:309-318.

Tenenbaum JB, Kemp C, Griffiths TL, Goodman ND (2011) How to grow a mind: statistics, structure, and abstraction. Science 331:1279-1285.

Turk-Browne NB, Scholl BJ, Chun MM, Johnson MK (2009) Neural evidence of statistical learning: efficient detection of visual regularities without awareness. J Cogn Neurosci 21:1934-1945.

Vilares I, Howard JD, Fernandes HL, Gottfried JA, Kording KP (2012) Differential representations of prior and likelihood uncertainty in the human brain. Curr Biol 22:1641-1648.

Wei K, Körding K (2010) Uncertainty of feedback and state estimation determines the speed of motor adaptation. Front Comput Neurosci 4:1-9.

Wurtz RH (2015) Brain mechanisms for active vision. Daedalus 144:10-21.

Yin C, Wang H, Wei K, Körding KP (2019) Sensorimotor priors are effector dependent. J Neurophysiol 122:389-397.

Zeligman L, Zivotofsky AZ (2017) Back to basics: the effects of block vs. interleaved trial administration on pro- and anti-saccade performance. PLoS One 12:e0172485. 